\title{
Generalizing Allen's Theory of Time to Tree-Like Structures
}

\author{
Salih Durhan*, Guido Sciavicco ${ }^{\dagger}$, \\ * Middle East Technical University, Northern Cyprus Campus (TRNC) - Email: sdurhan@metu.edu.tr \\ $\dagger$ University of Murcia (Spain) - Email: guido@um.es
}

\begin{abstract}
Allen's Interval Algebra is one of the most prominent formalisms in the area of qualitative temporal (and, by extension, spatial) reasoning. However, its applications are naturally restricted to linear flows of time. While there is some recent work focused on studying relations between intervals (and also between intervals and points) on branching structures, there is no rigorous study of the first-order theory of branching time. In this paper, we approach this problem under a very general definition of time structures as tree-like lattices. Allen's representation theorem shows that meets is expressively complete for the class of all unbounded linear orders, and it is easy to see that it is also complete for the class of all linear orders. Here we prove that, surprisingly, meets remains complete for the class of all unbounded tree-like lattices, and we provide an easy axiomatization of the class of all unbounded tree-like lattices in the branching language. Then, we show that meets becomes incomplete in the class of all tree-like lattices; we give a minimal complete set of three relations for this case along with an axiomatization, which turns out to be particulary challenging to obtain.
\end{abstract}

Keywords-Representation Theorems, Branching Time.

\section{INTRODUCTION}

Allen's Interval Algebra (IA) is one of the most prominent formalisms in the area of qualitative temporal, but also spatial, reasoning. However, its applications are naturally restricted to linear flows of time. Recent work focused on integrating intervals and points to obtain a more general firstorder language for linear time [8], and to obtain suitable extensions of Allen's IA for branching time with and without points [16], [17]. Nonetheless, studying the algebra that emerges from an extended set of relations and its computational complexity is not enough to obtain a clear understanding of the first-order structure that underlies it, and, as witnessed by a famous debate concerning Allen's firstorder axiomatization of unbounded interval-based structures (see [14], [10]), this study is not always easy. In this work, we provide a throughout analysis of first-order structures for branching time under minimal hypothesis: we only assume that structures are tree-like lattices, and we study both the unbounded and the general case in terms of a suitable pure first-order language to express the relations between two intervals on a tree-like lattice in Ligozat's style [15]. We present a first-order axiomatization of such structures in

The authors acknowledge the support from the Spanish Project TIN1239353-C04-01 and the Spanish fellowship program 'Ramon y Cajal' RYC2011-07821 (G. Sciavicco). similar terms to the original Allen's representation theorem in the unbounded case, proving that the sole relation meets remains complete. Then we prove that this is no longer the case when structures are not necessarily unbounded, and meets becomes incomplete. We present a simple complete set of three relations, and we give an axiomatization in this case as well. This problem was left open in [16] (Remark 5 ), and it turns out to be particularly challenging.

Various representation theorems exist in the literature for languages that include interval relations only on linear time: van Benthem [19], over rationals and with the interval relations during and before, Allen and Hayes [2], for the unbounded case without point intervals and for the relation meets, Ladkin [13], for point-based structures with a quaternary relation that encodes meeting of two intervals, Venema [21], for structures with the relations starts and finishes, Goranko, Montanari, and Sciavicco [11], that generalizes the results for structures with meets and met-by, Bochman [5], for point-interval structures, and Coetzee [7] for dense structure with overlaps and meets. Branching models of time which can be modeled by tree-like structures are of special interest for temporal reasoning at all levels, since they allow for representing indeterministic aspects of systems, scenarios, and planning tasks. At the modal logic level branching time models have been studied in depth in the recent past. Originated by philosophical logic [18], where branching time logics have been studied for analysis of indeterminism, causality, and action-theoretical concepts, branching time (point-based) logics such as CTL, CTL*, or ATL (see, e.g. [4], [9]) have been proposed as specification languages and, mainly, for model-checking purposes. On the other hand, as far as modal branching time interval-based logics are concerned, the literature is much more scarce; we mention here a future-only branching time version of Propositional Neighborhood Logic studied in [6]. Tree-like structures are a natural choice for modeling temporal aspects of events. For example, in [20] events are defined as closed interval in branching time, and branching structures are exploited to model the different courses that the world might take. The underlying idea is to identify an event with the set of its occurrences in time. An event may occur in many branches (actually an event is said to occur in a branch if and only if it is completely contained in that branch), and a close analysis of this model of time immediately allows 


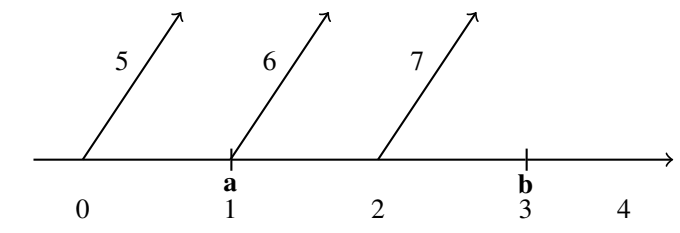

\begin{tabular}{|c|c|c|}
\hline Region & & Semantics \\
\hline$x 0[a, b]$ & $\Leftrightarrow$ & $x<a$ \\
\hline$x 1[a, b]$ & $\Leftrightarrow$ & $x=a$ \\
\hline$x 2[a, b]$ & $\Leftrightarrow$ & $a<x<b$ \\
\hline$x 3[a, b]$ & $\Leftrightarrow$ & $x=b$ \\
\hline$x 4[a, b]$ & $\Leftrightarrow$ & $x>b$ \\
\hline$x 5[a, b]$ & $\Leftrightarrow$ & $x \| a$ \\
\hline$x 6[a, b]$ & $\Leftrightarrow$ & $a<x \wedge(a<y \leq b \rightarrow y \| x)$ \\
\hline$x 7[a, b]$ & $\Leftrightarrow$ & $a<x \wedge \exists y(a<y<x \wedge a<y<b)$ \\
\hline
\end{tabular}

Table I

PICTORIAL REPRESENTATION AND NOTATION OF THE 8 REGIONS ARISING FROM AN INTERVAL.

one to realize how Allen's relations naturally emerge in this extended context. Also, in the area of automated planning it can be argued that branching time should be chosen as the correct model; as a matter of fact, planning tasks can be modeled as Kripke-like labeled graphs, which naturally unwind to be represented as (potentially unbounded) treelike structures.

Apart from the intrinsic interest in generalizing Allen's results to branching models of time, these results give a concrete answer to the question: When is a given abstract relational structure an interval-structure based on a branching model of time? Being able to answer this question is a key step in constraint satisfaction problems over branching models of time. For example, consider a scheduling problem in which there are two processes which are scheduled to start together and after a certain point they will be running in parallel. Each of these two processes may have their own sub-processes with various scheduling requirements. The whole system then is best modeled in a branching model of time and if one wants to check whether a given schedule is consistent one needs to know, among other things, that the abstract relational algebra described by the schedule is indeed based on a branching model of time.

\section{PREliminaries AND Notation}

Let $(\mathcal{T},<)$ be a partial order, whose elements are generally denoted by $a, b, \ldots, x, y, \ldots$. In the following, we shall use $a \| b$ to denote that $a$ and $b$ are incomparable w.r.t. the ordering relation $<$. A partial order $(\mathcal{T},<)$, often denoted by $\mathcal{T}$, is a future branching model of time if and only if for all $a, b \in \mathcal{T}$ there is a greatest lower bound of $a$ and $b$ in $\mathcal{T}$, and, if $a \| b$ then there exists no $c \in \mathcal{T}$ such that $c>a$ and $c>b$. Allen's representation theorem [1] works for the class Unb of all unbounded (i.e., such that every point has a successor and a predecessor) linearly ordered sets, and it can be immediately generalized for the class All of all linearly ordered sets [8]. Here, we are interested in: (i) the class TUnb of all unbounded future branching models of time, which immediately generalizes Unb, and (ii) the class TAll of all future branching models of time, which, symmetrically, generalizes All.

An interval in $\mathcal{T}$ is a pair $[a, b]$ where $a<b$, and $[a, b]=\{x \in \mathcal{T}: a \leq x \leq b\}$. Following Allen and Hayes [3], we adopt the so-called strict interpretation by asking that intervals with coincident endpoints are excluded. The latter (i.e., the points) can be recovered in an extended point-interval structure and theory, as it has been recently done in the case of linear time [8]. Now, let $\mathcal{I}(\mathcal{T})$ be the set of all intervals $[a, b]$ of $\mathcal{T}$. There are 24 basic relations (including equality) among any two intervals of $\mathcal{T}$, as described, for example, in [16], 13 of which come from Allen's IA over a linear order. As opposed to naming these relations with letters (which come from the initials of verbal description of the relation, such as $m$ to denote that two intervals meet) we shall use a more systematic notation, which is essentially a generalization of the one introduced in [15] and extended in [8]. If $[a, b] \in \mathcal{I}(\mathcal{T})$, then each point $x \in \mathcal{T}$ falls into exactly one of the 8 regions depicted in Tab. I. Accordingly, we say that the position of $x$ with respect to $[a, b]$ is $n$ (denoted by $x[a, b]$ ), where $n \in\{0, \ldots, 7\}$, if $x$ falls into region $n$, as in Tab. I.

It is easier to see the meaning of these positions over linear orders. If $\mathcal{T}$ is a linear order, then we only have the positions $0, \ldots, 4$ (see [15]). These positions describe whether $x<a, x=a, a<x<b, x=b$ or $b<x$ as can be seen below.

\begin{tabular}{lllll}
\hline & $\mathbf{b}$ & & & \\
\hline & $\mathbf{a}$ & 2 & $\mathbf{b}$ & \\
1 & & 2 & 3 & 4
\end{tabular}

Of course, when $\mathcal{T}$ is a future branching model of time there are more possible positions for $x$ with respect to a given interval $[a, b]$, and each of these are described in Tab. I. To describe a relation $\operatorname{Ir} J$ between intervals $I$ and $J$ we will use the position of the endpoints of $J$ with respect to $I$. In the case when $\mathcal{T}$ is a linear order, all positions are $0, \ldots, 4$ and each of Allen's relations are represented by $I n m J$, where $n, m \in\{0,1,2,3,4\}$, and $n$ is the position of the left endpoint of $J, m$ is the position of the right endpoint of $J$ with respect to $I$. If we take two equal intervals $I=[a, b]$ and $J=[c, d]$, then the position of the left endpoint $J$ with respect to $I$ is 1 and the position of the right endpoint $d$ of $J$ with respect to $I$ is 3 . Therefore equality is represented by $I 13 J$. As another example consider Allen's relations meets, take $I=[a, b], J=[c, d]$ and suppose that $I$ meets $J$. Then $c=b$ and so the postion of the left endpoint of $J$ with respect to $I$ is 3 . Since $b=c<d$, the position of the right 


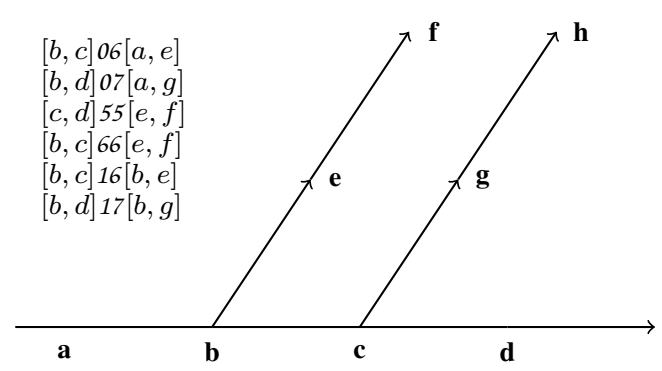

Relation Semantics

$\begin{array}{lll}{[a, b] 06[c, d]} & \Leftrightarrow & c<a<d \wedge \forall x(a<x \leq d \rightarrow x \| b) \\ {[a, b] 07[c, d]} & \Leftrightarrow & c<a<d \wedge d \| b \wedge \exists x(a, c<x<b, d) \\ {[a, b] 16[c, d]} & \Leftrightarrow & c=a \wedge \forall x(c<x \leq d \rightarrow x \| b) \\ {[a, b] 17[c, d]} & \Leftrightarrow & c=a \wedge d \| b \wedge \exists x(a, c<x<b, d) \\ {[a, b] 55[c, d]} & \Leftrightarrow & c \| a \\ {[a, b] 66[c, d]} & \Leftrightarrow & a<c \wedge \forall x(a<x \leq b \rightarrow x \| c) \\ {[a, b] 77[c, d]} & \Leftrightarrow & a<c \wedge b \| c \wedge \exists x(a<x<b, c)\end{array}$

$[a, b] 05[c, d] \Leftrightarrow a<c \wedge \forall x(c<x \leq d \rightarrow x \| a)$

$[a, b] 05^{e}[c, d] \quad \Leftrightarrow \quad a<c \wedge a \| d \wedge \exists x(c<x<d \wedge x<a)$

$[a, b] 27[c, d] \quad \Leftrightarrow \quad a<c<b \wedge \forall x(c<x \leq d \rightarrow x \| b)$

$[a, b] 27^{e}[c, d] \quad \Leftrightarrow \quad a<c<b \wedge d \| b \wedge \exists x(a, c<x<b, d)$

Table II

DETERMINED NON-LINEAR RELATIONS (TOP OF THE TABLE) PLUS THEIR PICTORIAL REPRESENTATION, AND UNDETERMINED NON-LINEAR RELATIONS (BOTTOM OF THE TABLE).

endpoint of $J$ with respect to $I$ is 4 . So the relation meets is represented by $I 34 J$.

Now, suppose $\mathcal{T}$ is a future branching model of time, and take $I=[a, b], J=[c, d]$, where $I, J \in \mathcal{I}(\mathcal{T})$. Moreover, let $n$ and $m$ be the positions of $c$ and $d$ with respect to the interval $[a, b]$. If both $n, m \in\{0, \ldots, 4\}$, then the pair $(n, m)$ uniquely describes the relation between $I$ and $J$, and we call these linear relations. Otherwise, the relation is called non-linear, and these require more careful consideration. Suppose the intervals $I, J$ stand in some nonlinear relation. For the cases described in Tab. II (top of the table), called determined non-linear relations, again the pair $(n, m)$ uniquely determines the relation between $I=[a, b]$ and $J=[c, d]$, which we denote by $\operatorname{InmJ}$. On the other hand, when $(n, m)$ is equal to $(0,5)$ or $(2,7)$ (see Tab. II, bottom of the table) there are two possible relations between $I$ and $J$. To resolve this issue we introduce a specific notation for these undetermined non-linear relations in order to distinguish the possible cases, namely we use InmJ or $I n m^{e} J$. Notice that in [8], an ontology which covers both intervals and points is considered and therefore on top of relations among intervals there are interval-point relations and point-point relations. To distinguish relations among intervals, the notation $n m_{i i}$ is used. We avoid this use of subscripts in our notation since we only consider relations among intervals.
For any relation $r$ between two intervals, its inverse relation, denoted by $\bar{r}$, is the relation

$$
I \bar{r} J \leftrightarrow J r I .
$$

In the cases of linear relations, the inverses of $00,01,02,23,22,12$ are $44,34,24,03,04,14$, respectively. Note that the linear relation 13 is the equality of intervals, which will be denoted $=$, and it is the inverse of itself. Moreover, from the above description, it is easy to see that the inverses of $05,05^{e}, 27,27^{e}$ are $66,77,06,07$, respectively, and that 16,17 and 55 are the inverses of themselves. Since we deal exclusively with first-order properties, we can omit the relations that may be defined simply as the inverse of another relation. Hence, if $\mathcal{L}_{L I}$ is the interval language over linear models of time (i.e., Allen's language, which includes the six basic linear relations plus equality), we can define the interval language over future branching models of time, denoted by $\mathcal{L}_{B I}$, as $\mathcal{L}_{L I} \cup \mathcal{D}$, where $\mathcal{D}$ is the set of all and only determined non-linear relations (see Tab. II, top of the table). Given a future branching model of time $\mathcal{T}$, the structure $\mathcal{I}(\mathcal{T})$ will be considered as a $\mathcal{L}_{B I}$-structure where the relations $\mathrm{nm}$ are interpreted as we have described. Therefore, for every relation $n m \in \mathcal{L}_{B I}$, we have that $[a, b] n m[c, d]$ if and only if $n$ is the position of $c$ and $m$ is the position of $d$ with respect to $[a, b]$ (notice that our choice w.r.t. the relations that are included in the language allows us to avoid the problem that arises from undetermined non-linear relations).

Definition 1: Given a class of partial (resp., linear) orders $\mathcal{C}$, the theory of future branching time intervals (resp., of linear time intervals) over $\mathcal{C}$ is the set of all $\mathcal{L}_{B I}$-sentences (resp., $\mathcal{L}_{L I}$-sentences) which hold in every $\mathcal{I}(\mathcal{T})$ (resp., every $\mathcal{I}(\mathcal{T})$ where $\mathcal{T}$ is a linear order) that belong to the class $\mathcal{C}$, and it is denoted by $\Sigma_{B I}^{\mathcal{C}}$ (resp., by $\Sigma_{L I}^{\mathcal{C}}$ ). A future branching (resp., linear) interval structure is any $\mathcal{L}_{B I^{-}}^{\mathcal{C}}$ structure (resp., $\mathcal{L}_{L I}^{\mathcal{C}}$-structure) $\mathcal{I}$ such that $\mathcal{I} \models \Sigma_{B I}^{\mathcal{C}}$ (resp., $\mathcal{I}=\Sigma_{L I}^{\mathcal{C}}$ ). The theory $\Sigma_{B I}^{\text {TAll }}$ (resp., $\Sigma_{L I}^{\text {All }}$ ) is simply denoted by $\Sigma_{B I}\left(\right.$ resp., $\left.\Sigma_{L I}\right)$.

Lemma 2: Let $\sigma$ be an $\mathcal{L}_{L I}$-sentence. Then

$$
\sigma \in \Sigma_{B I} \Leftrightarrow \sigma \in \Sigma_{L I} .
$$

Proof: The implication from left to right is clear since every linear order is a future branching model of time. For the other direction, let $\sigma \in \Sigma_{L I}$ and $\mathcal{T}$ a future branching model of time. For any linearly ordered $\mathcal{T}^{\prime} \subseteq \mathcal{T}$, $\sigma$ holds in $\mathcal{I}\left(\mathcal{T}^{\prime}\right)$. Since $\sigma$ involves only linear relations this implies that $\sigma$ holds in $\mathcal{I}(\mathcal{T})$ as well.

Given a language $\mathcal{L} \in\left\{\mathcal{L}_{L I}, \mathcal{L}_{B I}\right\}$, a subset $S \subseteq \mathcal{L}$, and a relation $r \in \mathcal{L}$, we say that $S$ defines $r$ over $\mathrm{C}$, denoted by $S_{\mathcal{C}} \rightarrow r$, if it is the case that $\Sigma_{L}^{\mathcal{C}}=(\operatorname{Ir} J) \leftrightarrow \varphi(I, J)$, for some $\mathcal{S}$-formula. Notice that definability is preserved from a class $\mathcal{C}$ to a smaller one $\mathcal{C}^{\prime}$. The set $S$ is complete over 
$\mathrm{C}$ if and only if $S \rightarrow_{\mathrm{C}} r$ for all $r \in L$; minimally complete over $\mathrm{C}$ (resp., maximally incomplete over $\mathrm{C}$ ) if and only if it is complete (resp., incomplete) over $\mathrm{C}$, and, every proper subset (resp., every strict superset) of $S$ is incomplete (resp., complete) over the same class. Allen and Hayes [2] proved that meets (i.e., 34 in our notation) is $\mathcal{L}_{L I}$-complete over Unb, and, as we have recalled, it immediately generalizes to prove that 34 is also $\mathcal{L}_{L I}$-complete over All.

\section{COMPleteness OVER TUnb}

In this section we prove that, perhaps surprisingly, Allen's relation 34 is $\mathcal{L}_{B I}$ complete over TUnb. Note that the fact that 34 is $\mathcal{L}_{L I}$-complete over Unb does not necessarily generalize to TUnb, as the relative definitions might change. Notice also that we state the result for the class TUnb while, in actuality, they hold for the super-class of TUnb that includes tree-lattices that are not necessarily unbounded to the left (only to the right). Proofs are stated in this slightly more general setting; the axioms themselves are identical for the two cases with the exception of the last one, which instead requires a (minimal) modification.

Lemma 3: For each $r \in \mathcal{L}_{L I},\{34\} \rightarrow$ TUnb $r$.

Proof: Let us proceed case-by-case by first presenting the defining formula for $r \in L I$, and, then, proving its correctness. In the rest of the proof, let $\mathcal{T}$ be an unbounded future branching model of time, $I=[a, b], J=[c, d]$, and let $\varphi$ denote the right-hand side of each equivalence. Note that once we prove that a relation is definable from 34, we can use it in defining other linear relations. We start by defining the relation 44 :

$$
(I 44 J) \leftrightarrow \quad \exists K(I 34 K \wedge K 34 J)
$$

In order to prove its correctness, suppose, first, that $\mathcal{I}(\mathcal{T}) \models$ $I 44 J$. Then, we have that $a<b<c<d$, and the interval $K=[b, c]$ witnesses the formula $\varphi(I, J)$. Second, assume that $\mathcal{I}(\mathcal{T})=\varphi(I, J)$. Then the interval $K$ witnessing $\varphi$ is $[b, c]$. Hence $a<b<c<d$, and we conclude that I44J. Next, we prove that we can define the equivalence between intervals:

$$
\begin{aligned}
(I=J) \leftrightarrow \quad & \forall K(K 34 I \leftrightarrow K 34 J) \wedge \\
& \forall K(I 34 K \leftrightarrow J 34 K) .
\end{aligned}
$$

Proving that $\mathcal{I}(\mathcal{T}) \models(I=J)$ implies $\varphi(I, J)$ is immediate. Assume, then, that $\mathcal{I}(\mathcal{T}) \models \varphi(I, J)$. If there is an interval $K$ such that $K 34 I$, then by the first conjunct of $\varphi$ we obtain $a=c$. If there is no such interval then $a$ is a lower bound of $\mathcal{T}$, and, since $\mathcal{T}$ is a future branching model of time, $a$ has to the smallest element of $\mathcal{T}$. Then $c$ also has to be the smallest element of $\mathcal{T}$ and hence $a=c$. Since $\mathcal{T}$ is unbounded from above there is $K$ such that $I 34$, which together with the second conjunct of $\varphi$ gives $b=d$, and, therefore $I=J$. We prove now that we can define 14 :

$$
\begin{aligned}
(I 14 J) \leftrightarrow \quad & \forall K(K 34 I \leftrightarrow K 34 J) \wedge \\
& \exists K \forall L((I 34 K) \wedge(K 34 L \leftrightarrow J 34 L)) .
\end{aligned}
$$

Suppose that $\mathcal{I}(\mathcal{T}) \models(I 14 J)$. Then $a=c$ and $b<d$; the first conjunct of $\varphi$ holds because $a=c$, and the second conjunct of $\varphi$ is witnessed by $K=[b, d]$. Assume, on the other hand, that $\mathcal{I}(\mathcal{T}) \models \varphi$. The first conjunct of $\varphi$ gives $a=c$ as in the previous lemma. Let $K=[e, f]$ witness the second conjunct of $\varphi$. Then, we have that $e=b$ and $f=d$, which leads to $b<d$ as required. As for the relation 03 we have:

$$
\begin{aligned}
(I 03 J) \leftrightarrow \quad & \forall K(I 34 K \leftrightarrow J 34 K) \wedge \\
& \exists K((K 34 I) \wedge(K 14 J)) .
\end{aligned}
$$

Again, suppose that $\mathcal{I}(\mathcal{T}) \models(I 03 J)$. Then, $c<a$ and $b=d$; the first conjunct of $\varphi$ holds since $b=d$, and the second conjunct of $\varphi$ is witnessed by $K=[c, a]$. Assume, now, that $\mathcal{I}(\mathcal{T}) \models \varphi$. By the first conjunct of $\varphi$ we obtain $b=d$, again using the assumption that each branch of $\mathcal{T}$ is unbounded. Let $K=[e, f]$ witness the second conjunct of $\varphi$. Then $f=a$ and $e=c$, and so we obtain $c<a$, as required. As for the relation 04 :

$$
(I 04 J) \leftrightarrow \exists K, L(K 34 I \wedge K 14 J \wedge I 34 L \wedge L 03 J) .
$$

Suppose, once more, that $\mathcal{I}(\mathcal{T}) \models(I 04 J)$; then $c<a$ and $b<d$, so that $K=[c, a]$ and $L=[b, d]$ witness $\varphi$. Next, assume that $\mathcal{I}(\mathcal{T}) \models \varphi$, witnessed by $K=[e, f]$ and $L=$ $[g, h]$. The first conjunct of $\varphi$ gives $f=a$, and the second conjunct gives $e=c$. Therefore we get $c<a$. The third conjunct of $\varphi$ gives $g=b$ and the fourth conjunct gives $h=d$. Hence we have $b<d$. Finally, we prove that we can define 24:

$$
(I 24 J) \leftrightarrow \exists K, L(K 14 I \wedge K 34 J \wedge I 34 L \wedge L 03 J) .
$$

Suppose that $\mathcal{I}(\mathcal{T}) \mid=(I 24 J)$; then, we have that $a<c<$ $b<d$, so that $K=[a, c]$ and $L=[b, d]$ witness $\varphi$. Next, assume that $\mathcal{I}(\mathcal{T}) \models \varphi$, witnessed by $K=[e, f]$ and $L=$ $[g, h]$. The first conjunct of $\varphi$ gives $e=a$ and $f<b$. The second conjunct gives $f=c$, so we get $a<c<b$. The third conjunct of $\varphi$ gives $g=b$ and fourth conjunct gives $h=d$, so we obtain $a<c<b<d$, as required.

So far we have derived all the linear relations in $\mathcal{L}_{B I}$ from 34; now we can use all of them to derive the remaining (nonlinear) relations. To this end, we introduce a new relation that holds when $I$ and $J$ do not satisfy any linear relations, by imposing that:

$$
\operatorname{IbrJ} \leftrightarrow\left(\wedge_{r \in \mathcal{L}_{L I}} \neg(\operatorname{Ir} J)\right) \wedge\left(\wedge_{r \in \mathcal{L}_{L I}} \neg(J r I)\right) .
$$

Lemma 4: $\Sigma_{B I}^{\text {TUnb }}=I b r J$ if and only if $b \| d$. 
(1) $\quad \forall I, J((\exists K(K 34 I \wedge K 34 J)) \rightarrow \forall K(K 34 I \rightarrow K 34 J))$

(2) $\forall I, J((\exists K(I 34 K \wedge J 34 K)) \rightarrow \forall K(I 34 K \rightarrow J 34 K))$

(3) $\quad \forall I(\neg I<I) \wedge \forall I, J(I<J \rightarrow J \nless I) \wedge \forall I, J, K((I<J \wedge J<K) \rightarrow I<K))$

(4) $\forall I, J \exists K(K \leq I \wedge K \leq J \wedge \forall L((L \leq I \wedge L \leq J) \rightarrow L \leq K)))$

(5) $\quad \forall I, J, K((I \| \bar{J} \wedge I \not \bar{J}) \rightarrow \neg(I<\bar{K} \wedge J<\bar{K}))$

(6) $\forall I \exists K(I 34 K) \wedge \forall I \exists K(K 34 I)$

Table III

AXIOMATIZATION OF TUnb IN THE LANGUAGE $L_{B I}$ (WHERE THE UNDERLINED CONJUNCT IS ADDED - IT MUST BE ELIMINATED TO AXIOMATIZE THE SUPER-CLASS OF TUnb THAT INCLUDES POSSIBLY LEFT-BOUNDED TREE-LIKE LATTICES).

Proof: Suppose that $\mathcal{I}(\mathcal{T})=I b r J$; we want to show that $b \| d$. Since $I$ and $J$ do not satisfy any linear relation, we have that either $a\|c, b\| c, a \| d$ or $b \| d$. If $a \| c$, since $a<b$ and $c<d$, we get $b \| d$ because $\mathcal{T}$ is future branching. If $b \| c$, since $c<d$, we get, again, $b \| d$. Likewise, if $a \| d, a<b$ leads to $b \| d$. As for the other direction, it is clear that if $b \| d$ then $I$ and $J$ do not satisfy any linear relation.

Lemma 5: For each $r \in \mathcal{L}_{B I} \backslash \mathcal{L}_{L I},\{34\} \rightarrow_{\text {TUnb }} r$.

Proof: As before, we proceed incrementally case-bycase, and, once again, let $\mathcal{T}$ be an unbounded future branching model of time, $I=[a, b], J=[c, d]$, and let $\varphi$ denote the right-hand side of each equivalence. Let us start by proving that 17 is definable:

$$
\begin{aligned}
(I 17 J) \leftrightarrow \quad & (I b r J) \wedge \forall K(K 34 I \leftrightarrow K 34 J) \wedge \\
& \exists K(K 14 I \wedge K 14 J) .
\end{aligned}
$$

To prove its correctness, suppose, first, that $\mathcal{I}(\mathcal{T}) \models$ $(I 17 J)$. Then we have that $a=c, b \| d$, and there exists $e$ such that $a<e<b$ and $c<e<d$. The first conjunct of $\varphi$ is satisfied since $b \| d$. The second conjunct of $\varphi$ is satisfied since $a=c$. The third conjunct of $\varphi$ is witnessed by the interval $K=[a, e]$. Next, assume that $\mathcal{I}(\mathcal{T}) \models \varphi$. By the first conjunct of $\varphi$ we get $b \| d$. The second conjunct of $\varphi$ gives us $a=c$. Let $K=[e, f]$ witness the third conjunct of $\varphi$; then $e=a=c, f<b$, and $f<d$. Hence, we have that $f$ satisfies $a<f<b$ and $c<f<d$; therefore, I17J as we wanted. Now, we define 16 :

$$
(I 16 J) \leftrightarrow \quad(I b r J) \wedge \forall K(K 34 I \leftrightarrow K 34 J) \wedge \neg(I 17 J) .
$$

Suppose, again, that $\mathcal{I}(\mathcal{T}) \models(I 16 J)$. Then $a=c$, and $x \| b$ whenever $c<x \leq d$. In particular $b \| d$ and so the first conjunct of $\varphi$ holds. The second conjunct of $\varphi$ is satisfied since $a=c$. The third conjunct of $\varphi$ is clearly satisfied. Next, assume that $\mathcal{I}(\mathcal{T}) \mid=\varphi$. Just as in the previous case the first and second conjuncts of $\varphi$ give $b \| d$ and $a=c$. The third conjunct of $\varphi$ gives $x \| b$ whenever $c<x \leq d$ and we obtain $I 16 J$. As for 55 :

$$
(I 55 J) \leftrightarrow \quad \exists K, L(K 34 I \wedge L 34 J \wedge K 16 L) .
$$

Suppose that $\mathcal{I}(\mathcal{T}) \models(I 55 J)$. Then $a \| c$, and since $\mathcal{T}$ is a future branching model of time we can take a greatest lower bound $e$ of $a$ and $c$. Then the intervals $K=[e, a]$ and $L=[e, c]$ witness $\varphi$. Next, assume that $\mathcal{I}(\mathcal{T}) \models \varphi$ witnessed by $K=[e, f]$ and $L=[g, h]$. Then $f=a$ and $h=c$. Since $K 16 L$, we get $a \| c$ as required. Now, as for 66:

$$
(I 66 J) \leftrightarrow \quad \exists K(K 16 I \wedge K 34 J) .
$$

Once again, suppose $\mathcal{I}(\mathcal{T}) \models(I 66 J)$. Then $a<c$ and $x \| c$ whenever $a<x \leq b$, and the interval $K=[a, c]$ witnesses $\varphi$. Next, assume that $\mathcal{I}(\mathcal{T})=\varphi$ witnessed by $K=[e, f]$. Then $e=a$ and $f=c$, and hence $a<c$. Since $[a, c] 16[a, b]$, we have that $x \| c$ whenever $a<x \leq b$. Therefore, we get I66J. Now, we can define 77:

$$
(I 77 J) \leftrightarrow \exists K(K 17 I \wedge K 34 J)
$$

Suppose that $\mathcal{I}(\mathcal{T}) \models(I 77 J)$. Then $a<c, b \| c$ and there is $x$ with if $a<x<b, c$. The greatest lower bound of $b$ and $c$ is greater than $a$ and the interval $K=[a, c]$ witnesses $\varphi$. Next, assume that $\mathcal{I}(\mathcal{T}) \mid=\varphi$ witnessed by $K=[e, f]$. Then, $e=a, f=c, b \| c$ and there is $x$ such that $a<x<b, c$. Therefore $I 77 J$. Towards an end, we focus on 06 :

$$
(I 06 J) \leftrightarrow \quad \exists K(K 16 I \wedge K 03 J)
$$

Again, suppose $\mathcal{I}(\mathcal{T}) \models(I 06 J)$. Then $c<a<d$ and if $a<x \leq d$ then $x \| b$. It is easy to see that the interval $K=$ $[a, d]$ witnesses $\varphi$. Next, assume that $\mathcal{I}(\mathcal{T}) \models \varphi$ witnessed by $K=[e, f]$. Then $e=a, f=d$, and moreover $a<c<$ $d$. Since $K 16 I$, we also have $x \| b$ whenever $a<x \leq d$. Therefore $I 06 J$. Finally, we define 07 :

$$
(I 07 J) \leftrightarrow \quad \exists K(K 17 I \wedge K 03 J) .
$$

Suppose $\mathcal{I}(\mathcal{T}) \models(I 07 J)$. Then $c<a<d, d \| b$ and there is $x$ with if $a, c<x<b, d$. The greatest lower bound of $b$ and $d$ is greater than $a$ and hence $K=[a, d]$ witnesses $\varphi$. Next, assume that $\mathcal{I}(\mathcal{T}) \models \varphi$ witnessed by $K=[e, f]$. Then $e=a, f=d$, and we have $c<a<d, b \| d$. Since $K 17 I$, the greatest lower bound of $d$ and $b$ is greater than $a$, and therefore $I 07 \mathrm{~J}$. 
Theorem 6: The set $\{34\}$ is minimally $L_{B I}$-complete on TUnb.

Now we will provide an axiomatization for $\Sigma_{B I}^{\mathrm{TUnb}}$. As we have mentioned, the provided axiomatization generalizes Allen's set of first-order conditions for an abstract structure to be interpreted as an unbounded branching model of time (as opposed to an unbounded linear order). First, let us introduce two new relations, defined using only 34 , which make the exposition and the axioms clearer.

$$
I \sim J \leftrightarrow \forall K(K 34 I \leftrightarrow K 34 J) .
$$

Note that $\sim$ is an equivalence relation for obvious reasons, regardless of the semantics of 34 . Of course, what we are aiming towards with the semantics of interval structures is that $I \sim J$ if and only if $I$ and $J$ have the same starting point. However, this is not immediate. As a matter of fact, we may possibly have two intervals with different starting points which are not met by any other interval. Now, we introduce a new definable (again using 34 only) relation, which is going to be used to define a partial ordering on the starting points of intervals:

$$
I<J \leftrightarrow \exists K(K \sim I \wedge K 34 J) .
$$

Let $\leq$ be a shorthand for the formula $(I<J \vee I=J)$, and let $I \| J$ be a shorthand for $(I \nless J \wedge J \nless I)$.

Lemma 7: The relation $<$ induces a well-defined relation on the set of equivalence classes of $\sim$. That is, if $I<J$, $I \sim I^{\prime}$ and $J \sim J^{\prime}$ then $I^{\prime}<J^{\prime}$.

Proof: Suppose $I<J, I \sim I^{\prime}$ and $J \sim J^{\prime}$. Let $K$ be such that $K \sim I$ and $K 34 J$. Since $\sim$ is an equivalence relation, $K \sim I^{\prime}$ and since $J \sim J^{\prime}$ we have $K 34 J^{\prime}$. Therefore $I^{\prime}<J^{\prime}$.

Again, note that the above lemma is independent from the semantics of the relation 34 .

Now, the first two axioms in Tab. III are based on the intuition that every interval should have a unique starting point and a unique end point. Axiom (3) expresses the idea that $<$ is a partial ordering, and, to impose that the partial ordering induced by $<$ on the equivalence classes of $\sim$ has greatest lower bounds (so it is future branching) and that it is unbounded from above we use Axiom (4), (5), and (6). The second conjunct of Axiom (6), underlined, can be added to obtain a representation theorem for TUnb, or eliminated to obtain a representation theorem for the super-class of TUnb of tree-like lattices unbounded to the right and not necessarily unbounded to the left.

Theorem 8: Let $\mathcal{I}$ be an $\mathcal{L}=\{34\}$-structure satisfying the axioms from (1) to (6) (including the last conjunct) in Tab. III. Then, there is an unbounded future branching model of time $\mathcal{T}$, definably interpretable in $\mathcal{I}$, such that $\mathcal{I}$ is isomorphic to $\mathcal{I}(\mathcal{T})$.

Proof: Let $\mathcal{T}=\mathcal{I} / \sim$, the set of equivalence classes of $\sim$ over $\mathcal{I}$. By Lemma $7,<$ induces a well-defined relation on $\mathcal{T}$ which we also denote by $<$. Note that $(\mathcal{T},<)$ is definably interpretable in $\mathcal{I}$ and moreover by Axiom (3), (4), (5), and (6), it is an unbounded future branching model of time. Now, for $\mathcal{L}=\{34\}$, we consider the set of intervals over $\mathcal{T}$ as a $\mathcal{L}$-structure where 34 is interpreted in the natural way, and define:

$$
\begin{aligned}
f: \mathcal{I} \rightarrow \mathcal{I}(\mathcal{T}) & \\
f(I) & \mapsto[I / \sim, J / \sim]
\end{aligned}
$$

where $J \in \mathcal{I}$ with $I 34 J$. Note that $J / \sim$ is independent of the choice of $J$. If $J^{\prime}$ is any other interval such that $I 34 J^{\prime}$, then by (1) we have $J \sim J^{\prime}$. Moreover, the existence of such $J$ is guaranteed by Axiom (6). We claim that $f$ is an isomorphism of $\mathcal{L}$-structures. Suppose that $f(I)=f(J)$. Then $I / \sim=J / \sim$ and there is $K$ such that $I 34 K$ and $J 34 K$. Using Axiom (1) and (2) it is easy to see that $I=J$. Note that $=$ is interpreted in $\mathcal{I}$ by the definition provided in Lemma 3. So $f$ is one-to-one. Let $[I / \sim, J / \sim] \in \mathcal{I}(\mathcal{T})$. Then $I / \sim<J / \sim$, and by the definition of $<$ there is $K \in \mathcal{I}$ such that $K \sim I$ and $K 34 J$. Therefore $f(K)=$ $[I / \sim, J / \sim]$ and so $f$ is onto. Finally, we need to show that $f$ respects the relation 34. Take $I, J \in \mathcal{I}$ such that $I 34 J$ and $f(I)=[I / \sim, K / \sim], f(J)=[J / \sim, L / \sim]$. Then $K$ is any interval such that $I 34 K$. Since we also have $I 34 J, K \sim J$ by (1) and hence $f(I) 34 f(J)$.

Theorem 9 (Representation Theorem): $\Sigma_{B I}^{\text {TUnb }}$ is axiomatized by Axiom (1) to (6) (including the last conjunct) in Tab. III, together with the definitions of all relations in $\mathcal{L}_{B I}$ from 34 .

Proof: It is clear that if $\mathcal{T}$ is an unbounded future branching model of time then (1) to (6) hold in $\mathcal{I}(\mathcal{T})$. Suppose that $\mathcal{I}$ is an $\mathcal{L}_{B I}$ structure satisfying (1) to (6), where each relation in $\mathcal{L}_{B I}$ is interpreted according to the definitions provided in Lemma 3 and Lemma 5. Then, by Theorem $8, \mathcal{I}$ is isomorphic to an interval structure $\mathcal{I}(\mathcal{T})$ and therefore $\mathcal{I}=\Sigma_{B I}^{\text {TUnb }}$.

\section{COMPleteness OVER TAll}

In this section, we consider the class TAll of all treelike lattices. In sharp contrast with Allen's original result and our results of the previous section, it turns out that the set $\{34\}$ is no longer complete in this case. Moreover, its incompleteness, paired with the presence of irregular tree-like lattices in the class (such as those lattices with an infinite number of unbounded branches and an infinite number of bounded ones) make the problem of devising an axiomatization of the class TAll particularly challenging. 
First, we prove that $\{34\}$ is incomplete for the class TAll. To this end, we recall the definition of surjective truth preserving relation.

Definition 10: Let $\mathcal{I}(\mathcal{T}), \mathcal{I}\left(\mathcal{T}^{\prime}\right)$ two concrete tree-like structures, and let $S \subseteq \mathcal{L}_{B I}$. A binary relation $\zeta \subseteq$ $\mathcal{I}(\mathcal{T}) \times \mathcal{I}\left(\mathcal{T}^{\prime}\right)$ is called a surjective $S$-truth preserving relation if and only if: (i) $\zeta$ respects the relations in $S$, i.e., if $\left(I, I^{\prime}\right),\left(J, J^{\prime}\right) \in \zeta$, then $r(I, J)$ if and only if $r\left(I^{\prime}, J^{\prime}\right)$ for every relation $r \in S$; (ii) $\zeta$ is total and surjective, i.e.: for every $I \in \mathcal{I}(\mathcal{T})$ ), there exist $I^{\prime} \in \mathcal{I}\left(\mathcal{T}^{\prime}\right)$ ) such that $\left(I, I^{\prime}\right) \in \zeta$ ), and for every $\left.I^{\prime} \in \mathcal{I}\left(\mathcal{T}^{\prime}\right)\right)$, there $I \in \mathcal{I}(\mathcal{T})$ ) such that $\left(I, I^{\prime}\right) \in \zeta$.

If $\zeta$ is a surjective $S$-truth preserving relation, we say that $\zeta$ breaks $r \notin S$ if and only if, there are $\left(I, I^{\prime}\right),\left(J, J^{\prime}\right) \in \zeta$ such that $r(I, J)$ but $\neg r\left(I^{\prime}, J^{\prime}\right)$. Now, it holds that if $\zeta$ is a surjective $S$-truth preserving relation between $\mathcal{I}(\mathcal{T})$ and $\mathcal{I}\left(\mathcal{T}^{\prime}\right)$, then for every first-order formula $\varphi$ in the language $\mathcal{L}_{B I}$ that uses only relations in $S$ it is the case that $\mathcal{I}(\mathcal{T}) \models$ $\varphi$ if and only if $\mathcal{I}\left(\mathcal{T}^{\prime}\right) \models \varphi$. Therefore in order to show that a given set $S$ is incomplete it suffices to show two structures $\mathcal{I}(\mathcal{T})$ and $\mathcal{I}\left(\mathcal{T}^{\prime}\right)$, along with a surjective $S$-truth preserving relation between them that breaks some $r \in L_{B I} \backslash S$.

Lemma 11: The sets $\{34,03\},\{=, 03\}$ and $\{=, 34\}$ are incomplete over TAll.

Proof: Consider, first, the case $\{34,03\}$. Let $\mathcal{T}=$ $\{a, b, c\}$ which is ordered by $a<b, a<c$ and $b \| c$. Let $f: \mathcal{I}(\mathcal{T}) \rightarrow \mathcal{I}(\mathcal{T})$ be the map which sends both $[a, b]$ and $[a, c]$ to $[a, b]$. Since there are no intervals over $\mathcal{T}$ which are related via 34 and 03 both relations are preserved, but, clearly, $=$ is not preserved and so $\{34,03\}$ is not complete. Next, consider the case $\{=, 03\}$. Let $\mathcal{T}=\{a, b, c, d\}$ which is ordered by $a<b<c, a<d$ and $d \| b$. Let $f: \mathcal{I}(\mathcal{T}) \rightarrow$ $\mathcal{I}(\mathcal{T})$ be the map which sends $[a, b]$ to $[a, d],[a, d]$ to $[a, b]$ and keeps all other intervals fixed. Since $f$ is one-to-one it preserves $=$. The only intervals related through 03 are $[a, c]$ and $[b, c]$, which are sent to themselves. So $f$ preservers 03 as well. However, $[a, b] 34[b, c]$ but $\neg([a, d] 34[b, c])$. So $\{=, 03\}$ is not complete. Finally, consider the case $\{=, 34\}$. Let $\mathcal{T}=\{a, b, c, d\}$ which is ordered by $a<b<c, a<d$ and $d \| b$. Let $f: \mathcal{I}(\mathcal{T}) \rightarrow \mathcal{I}(\mathcal{T})$ be the map which sends $[a, d]$ to $[a, c],[a, c]$ to $[a, d]$ and keeps all other intervals fixed. Since $f$ is one-to-one it preserves $=$. The only intervals related through 34 are $[a, b]$ and $[b, c]$, which are sent to themselves. So $f$ preserves 34 as well. However, $[b, c] 03[a, c]$ but $\neg([b, c] 03[a, d])$. So $\{=, 34\}$ is not complete.

Lemma 12: For each $r \in \mathcal{L}_{L I},\{=, 34,03\} \rightarrow$ TAll $r$.

Proof: As in the previous section, we proceed case-bycase by first presenting the defining formula for $r \in L I$, and, then, proving its correctness. In the rest of the proof, let $\mathcal{T}$ be a future branching model of time, $I=[a, b], J=[c, d]$, and let $\varphi$ denote the right-hand side of each equivalence.
Note that once we prove that a relation is definable from $\{=, 34,03\}$, we can use it in defining other linear relations. We start by defining the relation 44 :

$$
(I 44 J) \leftrightarrow \quad \exists K(I 34 K \wedge K 34 J) .
$$

Both the definition and its proof are identical to the corresponding case in Lemma 3. Next we define 14:

$$
\begin{array}{ll}
(I 14 J) \leftrightarrow \quad & \exists K(I 34 K \wedge K 03 J) \wedge \\
\cdot & \forall K(K 34 I \leftrightarrow K 34 J) .
\end{array}
$$

Suppose that $\mathcal{I}(\mathcal{T}) \models(I 14 J)$. Then $a=c$ and $b<d$; so the interval $K=[b, d]$ witnesses the first conjunct of $\varphi$ and the second conjunct of $\varphi$ holds since $a=c$. Assume, on the other hand, that $\mathcal{I}(\mathcal{T}) \models \varphi$. Since $\mathcal{T}$ is a future branching model of time, if a branch has a lower bound, then that lower bound is actually the smallest element of $\mathcal{T}$. This observation together with the second conjunct of $\varphi$ gives $a=c$. Now let $K=[e, f]$ witness the first conjunct of $\varphi$. Then $e=b$ and $f=d$, so $b<d$ as required. For the remaining cases, namely 04 and 24 , we have:

$$
\begin{aligned}
& (I 04 J) \leftrightarrow \exists K, L(K 34 I \wedge K 14 J \wedge I 34 L \wedge L 03 J), \\
& (I 24 J) \leftrightarrow \exists K, L(K 14 I \wedge K 34 J \wedge I 34 L \wedge L 03 J) .
\end{aligned}
$$

In both cases, the definitions and their proofs are identical to the ones presented in Lemma 3.

Lemma 13: For each $r \in \mathcal{L}_{B I} \backslash \mathcal{L}_{L I},\{=, 34,03\} \rightarrow$ All $r$.

Proof: All the definitions are the same with the ones presented in Lemma 5 and it is straightforward to check that all the proofs presented in Lemma 5 remain valid.

Now, Lemma 11, 12 and Lemma 13 give us the following theorem.

Theorem 14: The set $\{=, 34,03\}$ is minimally $L_{B I^{-}}$ complete on All.

Now, we present an axiomatization of $\Sigma_{B I}$, explicitly defined in Table IV. Axioms (1) to (5) of Table IV is the same as the ones presented in Table III. However, in the general case we will need more axioms since we need to capture the semantics more relations and the interplay between them. Note that we still make use of the relations $\sim$ and $<$ as defined in the previous section, and Lemma 7 still holds. We introduce a new relation

$$
I \approx J \leftrightarrow(I=J) \vee(I 03 J) \vee(J 03)
$$

and use $\approx$ also as a shorthand for the formula which defines it. It is clear that $I \approx I$ and if $I \approx J$ then $J \approx I$, regardless of the semantics. Axiom (6) in Table IV states the relation $\approx$ is transitive and hence it is an equivalence relation. So, the relation $\sim$ relates intervals with the same starting point and $\approx$ relates intervals with the same ending point. Axiom 


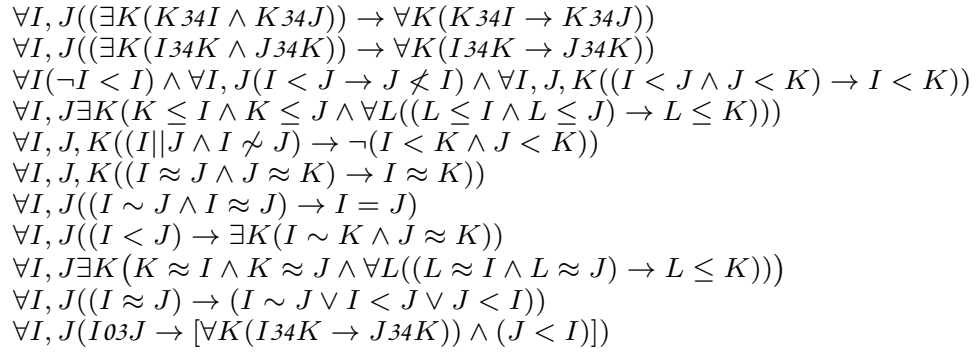

Table IV

Axiomatization of TAll IN THE LANGUAGE $L_{B I}$.

(7) states that intervals with the same starting and ending point are equal. Axiom (8) imposes that if the starting point if $I$ is less than the starting point if $J$ then there is an interval starting together with $I$ and ending together with $J$. Axiom (9) imposes greatest lower bounds to endpoints of intervals and Axiom (10) states the idea that the starting points of intervals which have the same endpoint should not be incomparable. Finally Axiom (11) gives the relation between 34 and 03 .

Theorem 15: Let $\mathcal{I}$ be an $\mathcal{L}=\{=, 34,03\}$-structure satisfying the above axioms from (1) to (11) in Table IV. Then, there is a future branching model of time $\mathcal{T}$, definably interpretable in $\mathcal{I}$, such that $\mathcal{I}$ is isomorphic to $\mathcal{I}(\mathcal{T})$.

Proof: Let $\mathcal{T}_{1}=\mathcal{I} / \sim$, be the set of equivalence classes of $\sim \operatorname{over} \mathcal{I}$. By Lemma $7,<$ induces a welldefined relation on $\mathcal{T}$ which we also denote by $<$. Note that $\left(\mathcal{T}_{1},<\right)$ is definably interpretable in $\mathcal{I}$ and it is a future branching model of time by Axioms (3), (4) and (5). Note also that in the general case $\mathcal{T}_{1}$ is not enough to capture all points, we need to also capture possible greatest points of branches. To do this we introduce the definable set $\mathcal{X}=\{I: \forall J \neg(I 34 J)\}$, which intuitively is the set of all cofinal intervals. Now let us define:

$$
\mathcal{T}=\mathcal{T}_{1} \biguplus(\mathcal{X} / \approx)
$$

We will equip $\mathcal{T}$ with an ordering, which extends the ordering $<$ on $\mathcal{T}_{1}$. Define a relation $R$ on $\mathcal{T}$ as follows:

$$
x R y \leftrightarrow\left\{\begin{array}{l}
x, y \in \mathcal{T}_{1} \text { and } x<y \text {, or } \\
x=(I / \sim) \in \mathcal{T}_{1}, y=(J / \approx) \in \mathcal{X} / \approx \\
\text { and there is } K \text { such that } K \sim I \text { and } K \approx J .
\end{array}\right.
$$

Since both $\sim$ and $\approx$ are equivalence relations, the relation $R$ is well-defined and moreover $(\mathcal{T}, R)$ is definably interpretable in $\mathcal{I}$. It is clear that $R$ is both irreflexive and antisymmetric. Now, suppose $x R y$ and $y R z$. Then either $x, y, z \in \mathcal{T}_{1}$ or $x, y \in \mathcal{T}_{1}$ and $z \in \mathcal{X} / \approx$. In the former case, we immediately get $x R z$ since $<$ is a partial ordering on $\mathcal{T}_{1}$. Therefore, suppose we are in the latter case with

$$
x=I / \sim, \quad y=J / \sim, \quad z=K / \approx .
$$

Then $I<J$ and there is $L$ with $L \sim J$ and $L \approx K$. This gives us $I<L$, and by Axiom (8) there exists $M$ with $M \sim$ $I$ and $M \approx L$. Since $\approx$ is an equivalence relation $M \approx K$, together with $M \sim I$ we get $(x R z)$. Hence, the relation $R$ is transitive, and so it is a partial order on $\mathcal{T}$ which extends the ordering $<$ on $\mathcal{T}_{1}$. From this point on, we also refer to $R$ as $<$. Axioms (9) and (10) ensure that $(\mathcal{T},<)$ is indeed a future branching model of time. Now, consider the set of intervals over $\mathcal{T}$ as a $\mathcal{L}$-structure where $\mathcal{L}=\{=, 34,03\}$ and the relations are interpreted in the natural way. Then, define:

$$
\begin{aligned}
f: \mathcal{I} & \rightarrow \mathcal{I}(\mathcal{T}) \\
f(I) & \mapsto\left[I / \sim, I_{e}\right]
\end{aligned}
$$

where $I_{e}=I / \approx$ when $I \in \mathcal{X}$, and $I_{e}=J / \sim$ where $I 34 \mathrm{~J}$, otherwise. Note that in either case we have that $(I / \sim)<I_{e}$, and that $f(I)$ is an interval over $\mathcal{T}$. We claim that $f$ is an isomorphism of $\mathcal{L}$-structures. Suppose that $f(I)=f(J)$. Then either both $I$ and $J$ are in $\mathcal{X}$ or they are both not in $\mathcal{X}$. In the latter case we obtain $I=J$ from Axioms (1) and (2) as in the previous section, and in the former case we obtain $I=J$ from Axioms (1) and (7). So $f$ is one-to-one. Let $[x, y] \in \mathcal{I}(\mathcal{T})$. Then either $x, y \in \mathcal{T}_{1}$ or $x \in \mathcal{T}_{1}$ and $y \in \mathcal{X} / \approx$. In the former case we have that

$$
x=(I / \sim)<y=(J / \sim),
$$

and, by the definition of $<$, there is $K \in \mathcal{I}$ such that $K \sim I$ and $K 34 J$. Therefore $f(K)=[x, y]$. In the latter case we have that

$$
x=(I / \sim)<y=(J / \approx),
$$

so there is $K$ with $K \sim I$ and $K \approx J$ and, hence, $f(K)=$ $[x, y]$. This shows $f$ is onto. Finally, we need to show that 
$f$ respects the relations 34 and 03 . Take $I, J \in \mathcal{I}$ such that $I 34 J$. Then $I \notin \mathcal{X}$, so $f(I)=[I / \sim, K / \sim]$ where $I 34 K$. Let $f(J)=\left[J / \sim, J_{e}\right]$. Then, by Axiom (1), we have $K \sim J$ and hence $f(I) 34 f(J)$. Next, take $I, J \in \mathcal{I}$ with $I 03 J$. By Axiom (11), either both $I$ and $J$ are in $\mathcal{X}$ or they are both not in $\mathcal{X}$. In the former case we have that

$$
f(I)=\left[I / \sim, K_{1} / \sim\right], \quad f(J)=\left[J / \sim, K_{2} / \sim\right]
$$

where $I 34 K_{1}$ and $J 34 K_{2}$. Using Axiom (11) again, we get $J 34 K_{1}$ as well. Therefore Axiom (1) gives us $K_{1} \sim K_{2}$. Using Axiom (11) one last time, we see $J<I$ and we get $f(I) 03 f(J)$. Now assume that both $I$ and $J$ are in $\mathcal{X}$. Then we have that

$$
f(I)=[I / \sim, I / \approx], \quad f(J)=[J / \sim, J / \approx] .
$$

Since $I 03 J$, we immediately obtain $I \approx J$. Moreover, by Axiom (11), we have that $J<I$, so that $f(I) 03 f(J)$.

Theorem 16 (Representation Theorem): $\Sigma_{B I}$ is axiomatized by Axioms (1) to (11) in Tab. IV, together with the definitions of all relations in $\mathcal{L}_{B I}$ from $\{=, 34,03\}$.

Proof: It is clear that if $\mathcal{T}$ is a future branching model of time then (1) to (11) hold in $\mathcal{I}(\mathcal{T})$. Suppose that $\mathcal{I}$ is an $\mathcal{L}_{B I}$ structure satisfying (1) to (11), where each relation in $\mathcal{L}_{B I}$ is interpreted according to the definitions provided in Lemma 12 and Lemma 13. Then, by Theorem 15, $\mathcal{I}$ is isomorphic to an interval structure $\mathcal{I}(\mathcal{T})$ and therefore $\mathcal{I} \models$ $\Sigma_{B I}^{\text {TUnb }}$.

\section{CONCLUSions}

Allen's Interval Algebra is one of the most prominent formalisms in the area of qualitative temporal and spatial reasoning. However, its applications are naturally restricted to linear flows of time. In the context of a rigorous study of the first-order theory of linear time, the most influential result is Allen's representation theorem [2] for the class of all unbounded linear orders (which immediately generalizes to the class of all linear orders), and the minimal completeness of the relation meets, which is enough to define all other interval relations. In this paper we aimed to generalize the theory to the branching case, by defining temporal structures as tree-like lattices. After defining a suitable branching language, for the class of all unbounded tree-like lattices we were able to prove that, perhaps surprisingly, meets remains complete; the consequent axiomatization turned out to be rather elegant and easy-to-prove complete. On the other hand, for the case of all tree-like lattices, meets becomes incomplete. We obtained a minimal complete set of branching relations, over which we defined a more complex axiomatization that we shown to be complete. These problems had been stated in [16].
Besides pure theoretical interest, a branching Interval Algebra is a useful tool in constraint satisfaction and planning, and we gave it a solid theoretical basis. Furthermore, there is a general interest in studying variants of Interval Algebra that not only deal with branching time, but also restrict the set of relations to coarser ones that correspond precisely to period relations as implemented in the standard SQL:2011 [12]. Our study goes precisely in this direction, and, possibly, towards defining the bases for a branching version of temporal databases.

\section{REFERENCES}

[1] J. Allen, "Maintaining knowledge about temporal intervals," Communications of the ACM, vol. 26, no. 11, pp. 832-843, 1983.

[2] J. Allen and P. Hayes, "A common-sense theory of time," in Proc. of the 9th International Joint Conference on Artificial Intelligence. Morgan Kaufmann, 1985, pp. 528-531.

[3] J. Allen and P. J. Hayes, "Short time periods," in Proc. of the 10th International Joint Conference on Artificial Intelligence, 1987, pp. 981-983.

[4] R. Alur, T. Henzinger, and O. Kupferman, "Alternating-time temporal logic," Journal of the ACM, vol. 49, no. 5, pp. 672713, 2002.

[5] A. Bochman, "Concerted instant-interval temporal semantics i: Temporal ontologies," Notre Dame Journal of Formal Logic, vol. 31, no. 3, pp. 403-414, 1990.

[6] D. Bresolin, A. Montanari, and P. Sala, "An optimal tableau for right propositional neighborhood logic over trees," in Proc. of the 15th International Symposium on Temporal Representation and Reasoning (TIME). IEEE, 2008, pp. 110-117.

[7] C. J. Coetzee, "Representation theorems for classes of interval structures," Master's thesis, Department of Mathematics, University of Johannesburg, 2009.

[8] W. Conradie, S. Durhan, and G. Sciavicco, "An integrated first-order theory of points and intervals: Expressive power in the class of all linear orders," in Proc. of the 19th International Symposium on Temporal Representation and Reasoning (TIME). IEEE, 2012, pp. 47 - 51.

[9] E. A. Emerson, "Temporal and modal logic," in Handbook of Theoretical Computer Science, Volume B: Formal Models and Sematics (B), 1990, pp. 995-1072.

[10] A. Galton, "Note on a lemma of Ladkin," Journal of Logic and Computation, vol. 6, no. 1, pp. 1-4, 1996.

[11] V. Goranko, A. Montanari, and G. Sciavicco, "Propositional interval neighborhood temporal logics," Journal of Universal computer science, vol. 9, no. 9, pp. 1137-1167, 2003.

[12] K. Kulkarni and J. Michels, "Temporal features in SQL:2011," SIGMOD Records, vol. 41, no. 3, pp. 34-43, 2012. 
[13] P. Ladkin, "The Logic of Time Representation," Ph.D. dissertation, University of California, Berkeley, 1978.

[14] — "Models of axioms for time intervals," in Proc. of the 6th National Conference on Artificial Intelligence. Morgan Kaufmann, 1987, pp. 234-239.

[15] G. Ligozat, "On generalized interval calculi," in Proc. of the 9th National Conference on Artificial Intelligence (AAAI 91), 1991, pp. 234-240.

[16] M. Ragni and S. Wölfl, "Branching Allen," in Proc. of the 4th International Conference on Spatial Cognition, ser. Lecture Notes in Computer Science, vol. 3343. Springer, 2004, pp. 323-343.

[17] A. Reich, "Intervals, points, and branching time," in Proc. of the 9th International Symposium on Temporal Representation and Reasoning (TIME). IEEE, 1994, pp. 121-133.

[18] R. Thomason, "Combination of tense and modality," in Handbook of Philosophical Logic, D. Gabbay and F. Guenthner, Eds., vol. II, 1984, pp. 135-165.

[19] J. van Benthem, The Logic of Time (2nd Edition). Kluwer Academic Press, 1991.

[20] F. van Kutshera, "Sebastian's strolls," Grazer Philosophisce Studien, no. 45, pp. 75-88, 1993.

[21] Y. Venema, "A modal logic for chopping intervals," Journal of Logic and Computation, vol. 1, no. 4, pp. 453-476, 1991. 